\title{
Deep Convolutional Neural Network
} Based on Computed Tomography Images for the Preoperative Diagnosis of Occult Peritoneal Metastasis in Advanced Gastric Cancer

\section{OPEN ACCESS}

Edited by:

Changqiang Wu,

North Sichuan Medical College, China

Reviewed by:

Zaiyi Liu,

Guangdong Provincial People's

Hospital, China

Shenghong Ju,

Southeast University, China

${ }^{*}$ Correspondence:

Jiankun Hu

hujkwch@126.com

Bin Song

songlab_radiology@163.com

${ }^{+}$These authors have contributed equally to this work

Specialty section: This article was submitted to Cancer Imaging and Image-directed Interventions, a section of the journal

Frontiers in Oncology

Received: 01 September 2020

Accepted: 12 October 2020

Published: 02 November 2020

Citation:

Huang Z, Liu D, Chen X, He D, Yu P,

Liu B, Wu B, Hu J and Song B (2020)

Deep Convolutional Neural Network

Based on Computed Tomography

Images for the Preoperative Diagnosis

of Occult Peritoneal Metastasis in

Advanced Gastric Cancer.

Front. Oncol. 10:601869.

doi: 10.3389/fonc.2020.601869

\author{
Zixing Huang ${ }^{1 \dagger}$, Dan Liu ${ }^{1 \dagger}$, Xinzu Chen ${ }^{2}$, Du He ${ }^{3}$, Pengxin $\mathrm{Yu}^{4}$, Baiyun $\mathrm{Liu}^{4}$, Bing Wu ${ }^{1}$, \\ Jiankun $\mathrm{Hu}^{2^{*}}$ and Bin Song ${ }^{{ }^{*}}$ \\ ${ }^{1}$ Department of Radiology, West China Hospital, Sichuan University, Chengdu, China, 2 State Key Laboratory of Biotherapy, \\ Department of Gastrointestinal Surgery and Laboratory of Gastric Cancer, Collaborative Innovation Center for Biotherapy, \\ West China Hospital, Sichuan University, Chengdu, China, ${ }^{3}$ Department of Pathology, West China Hospital, Sichuan University, \\ Chengdu, China, ${ }^{4}$ Institute of Advanced Research, Infervision, Beijing, China
}

We aimed to develop a deep convolutional neural network (DCNN) model based on computed tomography $(C T)$ images for the preoperative diagnosis of occult peritoneal metastasis (OPM) in advanced gastric cancer (AGC). A total of 544 patients with AGC were retrospectively enrolled. Seventy-nine patients were confirmed with OPM during surgery or laparoscopy. CT images collected during the initial visit were randomly split into a training cohort and a testing cohort for DCNN model development and performance evaluation, respectively. A conventional clinical model using multivariable logistic regression was also developed to estimate the pretest probability of OPM in patients with gastric cancer. The DCNN model showed an AUC of 0.900 (95\% Cl: 0.851-0.953), outperforming the conventional clinical model (AUC $=0.670,95 \% \mathrm{Cl}: 0.615-0.739 ; p<$ 0.001). The proposed DCNN model demonstrated the diagnostic detection of occult PM, with a sensitivity of $81.0 \%$ and specificity of $87.5 \%$ using the cutoff value according to the Youden index. Our study shows that the proposed deep learning algorithm, developed with CT images, may be used as an effective tool to preoperatively diagnose OPM in AGC.

Keywords: stomach neoplasms, peritoneal neoplasms, deep learning, tomography, $x$-ray computed, neural networks, computer

\section{INTRODUCTION}

According to the GLOBOCAN 2018 data, gastric cancer (GC) remains the fifth most common cancer and the third most deadly cancer worldwide (1). Peritoneal metastasis (PM) occurs in 53$66 \%$ of patients diagnosed with metastatic GC (2), especially in younger patients with advanced gastric cancer (AGC) (3). Patients with PM thus may be subject to late detection or even improper 
surgical treatment. Therefore, the early detection and diagnosis of PM in GC patients prior to surgery would be crucial for avoiding unnecessary resection and allow for optimal therapy selection in clinical practice (4-9).

Abdominal enhanced CT is considered the most common noninvasive modality of preoperative diagnosis in GC patients (5, 7, 9-11). Typical PM indications on CT images include omentum cake, extensive ascites, and parietal peritoneal thickening (12). Clinically, occult peritoneal metastasis (OPM) often refers to PM negativity on initial CT diagnosis that is revised to PM positivity following subsequent laparoscopy or surgery $(12,13)$. Due to the nature of OPM, it is often missed by radiologists when interpreting CT images alone, resulting in low detection sensitivity and diagnostic accuracy in AGC patients. It has been reported that approximately $16 \%$ of OPMs are missed on CT images $(12,14-16)$, even with multidisciplinary discussion $(12,13)$. MRI and PET/CT are considered second choices because they are less sensitive than abdominal enhanced CT in detecting peritoneal metastases (17-19). In addition, the costs of MRI and especially PET/CT are high. Recent advances in technology using laparoscopy have provided reliable preoperative methods to identify OPM in patients with AGC $(5,8-10,20,21)$. However, there are many medical concerns and adverse medical care issues due to its invasive and costly nature, and its application in appropriate patient selection remains controversial. Therefore, the development of a noninvasive method to facilitate the targeted diagnosis of OPM beyond conventional imaging is urgently needed.

Artificial intelligence (AI) technology, particularly deep learning, has shown remarkable progress in medical image interpretation (22-24). A typical deep learning approach, named convolutional neural network (CNN), is a novel and powerful tool for the image-based determination of complex relationships and has exhibited sophisticated performance for small feature detection and characterization (25-27). The literature has reported the use of CNNs in the detection and diagnosis of tumor diseases, such as prostate cancer, breast cancer, and lung cancer $(28,29)$, highlighting the value of deep learning in clinical practice.

We therefore aimed to develop a deep CNN (DCNN)-based model for the preoperative diagnosis of OPM in AGC patients and to compare its diagnostic performance with that of the conventional clinical model using logistic regression.

\section{MATERIALS AND METHODS}

This retrospective study was approved by the Biomedical Research Ethics Committee of West China Hospital of Sichuan University, and the requirement for informed consent was waived.

\section{Patients}

The study was carried out at Surgical Gastric Cancer Patient Registry of West China Hospital (id: WCH-SGCPR-2019-08). Patients were enrolled based on the following inclusion criteria:
(1) patients with AGC ( $\mathrm{cT} \geq 2$ ) diagnosed by endoscopy-biopsy and CT; (2) patients who received whole abdominal enhanced CT scan preoperatively with a venous-image slice thickness of $2 \mathrm{~mm}$; (3) patients without typical PM findings on CT, such as omental nodules or omental cake, extensive ascites, or irregular thickening with high peritoneal enhancement; and (4) patients with no other evidence of distant metastasis or other tumors. The exclusion criteria were as follows: (1) previous abdominal surgery; (2) previous abdominal malignancies or inflammatory diseases; (3) CT carried out more than 2 weeks before surgery; (4) poor stomach filling; (5) poor CT image quality due to artifacts; and (6) inability to visualize the primary lesion of gastric cancer on CT images. The selection process of patients included in this study is shown in Figure 1.

PM status confirmed: All patients were confirmed to have peritoneal metastasis by surgical or laparoscopic exploration. The laparoscopy procedure used here was a "Four-Step Procedure" of laparoscopic exploration for GC (30). During the procedure, the abdominal and peritoneal conditions were carefully examined. All suspicious peritoneal implants or ascites were sent for pathological biopsy or cytological examination. Existence of PM was determined using the American Joint Committee on Cancer guidelines in consensus between pathologists and surgeons.

\section{CT Image Acquisition}

Prior to CT examination, patients were requested to fast for at least $6 \mathrm{~h}$ and orally ingested $600-1000 \mathrm{~mL}$ water. Patients were first trained to hold their breath during scanning with the scan range covering the entire abdomen and then scanned using a 128-slice scanner (SOMATOM Definition AS+, Siemens Healthcare, Forchheim, Germany) and a dual-source CT system (Somatom Definition Flash, Siemens Healthcare, Forchheim, Germany) with the following parameters: tube voltage, $120 \mathrm{kV}$; amperage, $210 \mathrm{mAs}$; slice thickness, $2 \mathrm{~mm}$; slice interval, $2 \mathrm{~mm}$; field of view, 35-50 cm; matrix, $512 \times 512$; rotation time, $0.5 \mathrm{~s}$; and pitch 1.0. With a trigger threshold of the aorta reaching $170 \mathrm{HU}$, a three-phase scan was obtained in the precontrast phase, the arterial phase at the trigger, and the portal vein phase $30 \mathrm{~s}$ after the trigger. Following an unenhanced scan, $1.2-1.5 \mathrm{~mL} / \mathrm{kg}$ iodinated contrast agent [Iopamiro $(370 \mathrm{mg}$ $\mathrm{I} / \mathrm{mL}$ ), Shanghai Bracco Sine Pharmaceutical Corp Ltd, Shanghai, China] was injected intravenously at a flow rate of 2.5-3.0 mL/s using a high-pressure syringe (Medrad Stellant CT Injector System, Medrad Inc. Inianola, USA).

\section{Data Preparation}

Portal vein-phase CT images were first exported to ITK-SNAP software (version 2.2.0; www.itksnap.org) for manual segmentation. Gastric cancer lesions were then manually annotated by a radiologist (with 5 years of experience in gastroenterology imaging) and confirmed by another abdominal specialist (with 14 years of experience in gastroenterology imaging). Two radiologists reviewed all slices obtained from each patient, selected one slice with the largest tumor area and manually delineated the lesion to obtain the final regions of interest (ROIs) (Figure 2). The gastric lumen and artifacts were carefully avoided. 


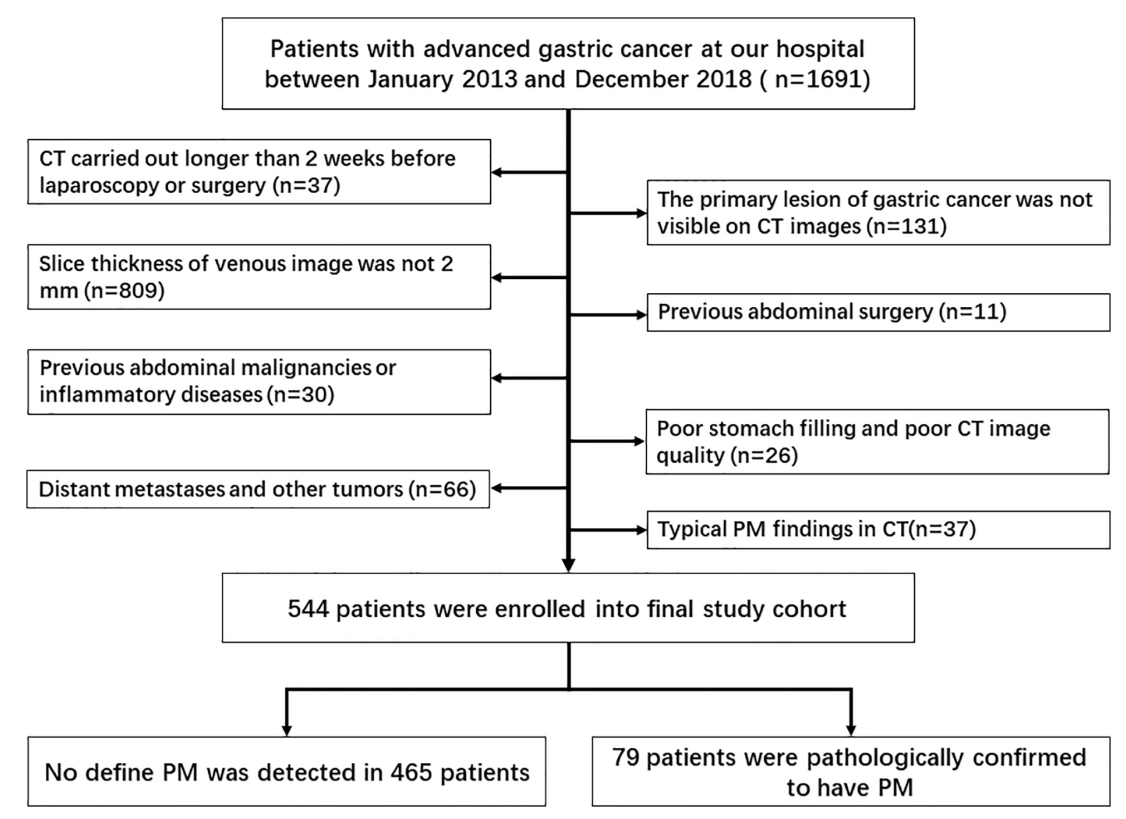

FIGURE 1 | Flow chart of the selection process for the included studies.

\section{Image Preprocessing}

We first converted the images into grayscale Joint Photographic Experts Group format based on each patient's CT images and the corresponding ROI masks. Before training the DCNN model, we applied data augmentation techniques to create new pseudoimages to expand the training sample size and enhance the model generalizability (31). Details of the data augmentation are explained in the Supplementary Material.

\section{DCNN Model}

A simple workflow scheme for the development of the DCNN model is shown in Figure 3. The backbone of the DCNN model employs Xception (32), which had been pretrained on the ImageNet database $(33,34)$. The main structure contains a Depthwise Convolution block and a Depthwise Separable Convolution designed within the block. Considering the class imbalance among the number of PM-positive cases, we utilized a stacking

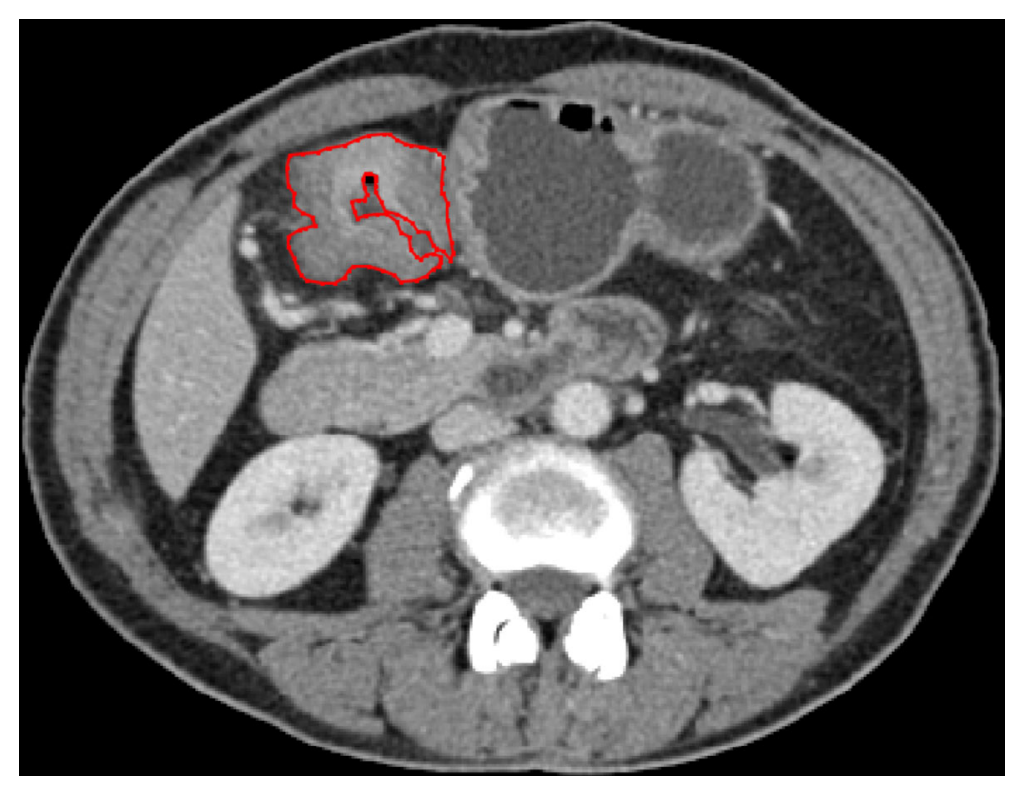

FIGURE 2 | ROI annotation (red line) on a representative CT image, with the largest area of the primary lesion drawn on the axial plane. 

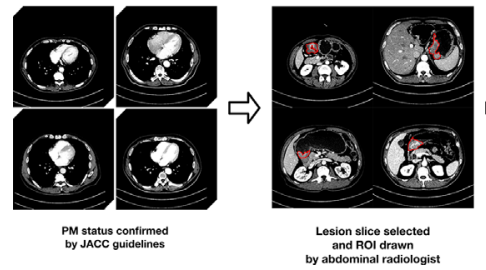
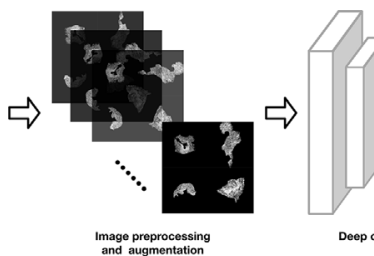

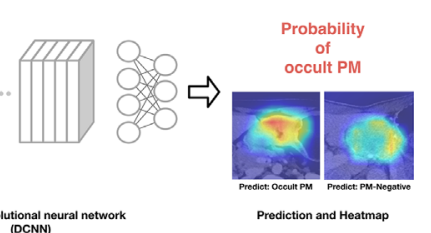

(DCNN)

FIGURE 3 | Proposed deep convolutional neural network (DCNN) workflow for OPM detection and prediction.

strategy to train the model (35). Model training details and the stacking strategy are presented in the Supplementary Material.

After the training was completed, the testing cohort was used as the input into the DCNN model, and the model performance was evaluated using receiver operating characteristic (ROC) analysis. Heatmaps generated by gradient-weighted class activation mapping (Grad-CAM) were applied to display activated areas of the presence of OPM predicted by the DCNN model.

\section{Clinical Model}

The preoperative characteristic features were applied to a multivariable logistic regression analysis to determine independent predictors of OPM. Backward stepwise selection was utilized based on the Akaike information criterion (AIC) (36). The clinical model was then developed based on the independent characteristic features and applied to the testing cohort. The diagnostic performance of the clinical model was assessed using ROC analysis. The cutoff for the ROC curve was determined by the Youden index.

\section{Statistical Analysis}

Considering the class imbalance in the testing cohort, we used bootstrapping $(n=1000)$ to calculate the $95 \%$ confidence intervals (CIs). A decision curve was plotted to evaluate model efficacy by quantifying the net benefits at different probability thresholds. Differences in continuous variables were analyzed with the independent t-test, and differences in categorical variables were analyzed with the chi-squared test. DeLong's test was used to compare the ROC curves of models. The generalizability of the DCNN model was evaluated in subgroups of age and sex using ROC curves. All statistical analyses were performed with R software (version 3.5.0; http://www.Rproject.org) and SPSS 22.0 (IBM, Armonk, NY, USA). A two-tailed $p$ value lower than 0.05 was considered statistically significant.

\section{RESULTS}

Finally, a total of 544 patients (age, 17-87 years; median age, 60 years), including 359 men and 185 women, were enrolled in this study. All patients were confirmed with advanced GC based on end-point diagnoses, among who 79 patients were confirmed as OPM-positive using laparoscopy, while the rest $(n=465)$ were defined as PM-negative. Table 1 shows the enrolled patients' demographic information. The determination of OPM presence is described in detail in the Supplementary Material. None of the patients were treated with neoadjuvant chemotherapy.

The entire cohort of 544 patients was randomly divided into a training set comprising 395 patients (58 OPM-positive and 337 PM-negative) and a testing set comprising 149 patients (21 OPM-positive and $128 \mathrm{PM}$-negative).

\section{Clinical Characteristics in the Training and Testing Cohorts}

As shown in Table 1, there were significant differences between lesion location and Borrmann type for the OPM-positive and PM-negative groups in the training cohort $(p<0.001)$. There was no significant difference in age, sex, carcinoembryonic antigen (CEA), or carbohydrate antigen 19-9 (CA19-9) between the OPM-positive and PM-negative groups in the entire cohort.

\section{Diagnostic Performance Measurements Clinical Model}

As shown in Table 2, multivariable logistic regression analysis identified the Location-L/L+D and Borrmann type as independent predictors $(p<0.05)$ for OPM positivity and PM negativity. A clinical model that incorporated the independent predictors was developed, and a ROC curve was created. The area under the ROC curve (AUC) was 0.670 (95\% CI: $0.615-$ 0.739 ). The sensitivity of the clinical model for the testing cohort was $85.7 \%$, with a specificity of $44.5 \%$ (Table 3 ).

\section{DCNN Model}

Along with the performance generated in the clinical model, Table 3 also shows the diagnostic performance of the DCNN model using the same testing cohort. In the bootstrapping validation, the DCNN model yielded an AUC of 0.900 (95\% CI: $0.851-0.953$ ), a sensitivity of $81.0 \%$ and a specificity of $87.5 \%$. Comparison of the ROC curves of the models suggested that the DCNN model significantly outperformed the clinical model $(\mathrm{p}<0.001)$. The ROC curves of the DCNN model and clinical model are shown in Figure 4.

\section{Stratified Analysis of Sex and Age}

Deep learning algorithms frequently suffer from issues of generalizability. To test the generalization ability of the proposed DCNN model, we performed stratification analysis 
TABLE 1 | Characteristics of patients in the training and testing cohorts.

\begin{tabular}{|c|c|c|c|c|c|c|c|c|c|c|}
\hline \multirow[t]{3}{*}{ Characteristic } & \multicolumn{4}{|c|}{ Training cohort $(\mathrm{N}=395)$} & \multirow[t]{3}{*}{$p$} & \multicolumn{4}{|c|}{ Validation cohort $(N=149)$} & \multirow[t]{3}{*}{$p$} \\
\hline & \multicolumn{2}{|c|}{ OPM Pos(N = 58) } & \multicolumn{2}{|c|}{ OPM Neg(N = 337) } & & \multicolumn{2}{|c|}{ OPM Pos(N = 21) } & \multicolumn{2}{|c|}{ OPM Neg(N = 128) } & \\
\hline & Mean & Std & Mean & Std & & Mean & Std & Mean & Std & \\
\hline \multirow[t]{2}{*}{ Age (years) } & 58.17 & 13.24 & 57.99 & 11.74 & 0.451 & 55.86 & 16.33 & 60.41 & 10.76 & 0.121 \\
\hline & $\mathrm{N}$ & $\%$ & $\mathrm{~N}$ & $\%$ & & $\mathrm{~N}$ & $\%$ & $\mathrm{~N}$ & $\%$ & \\
\hline Gender & & & & & 0.270 & & & & & 0.469 \\
\hline Male & 34 & 58.6 & 226 & 67.1 & & 12 & 57.1 & 87 & 68.0 & \\
\hline Female & 24 & 41.4 & 111 & 32.9 & & 9 & 42.9 & 41 & 32.0 & \\
\hline Location & & & & & 0.002 & & & & & 0.116 \\
\hline $\mathrm{U} / \mathrm{U}+\mathrm{M}$ & 12 & 20.7 & 68 & 20.2 & & 3 & 14.3 & 36 & 28.1 & \\
\hline $\mathrm{M} / \mathrm{M}+\mathrm{L}$ & 20 & 34.5 & 63 & 18.7 & & 6 & 28.6 & 18 & 14.1 & \\
\hline $\mathrm{L} / \mathrm{L}+\mathrm{D}$ & 18 & 31.0 & 181 & 53.7 & & 9 & 42.9 & 63 & 49.2 & \\
\hline$U+E$ & 1 & 1.7 & 11 & 3.3 & & 0 & 0.0 & 5 & 3.9 & \\
\hline Whole stomach & 7 & 12.1 & 14 & 4.2 & & 3 & 14.3 & 6 & 4.7 & \\
\hline Borrmann type & & & & & 0.000 & & & & & 0.009 \\
\hline Type 1, 2 & 48 & 82.8 & 194 & 57.6 & & 18 & 85.7 & 71 & 55.5 & \\
\hline Type 3, 4 & 10 & 17.2 & 143 & 42.4 & & 3 & 14.3 & 57 & 44.5 & \\
\hline CEA & & & & & 0.313 & & & & & 0.128 \\
\hline normal & 36 & 62.1 & 235 & 69.7 & & 18 & 85.7 & 88 & 68.8 & \\
\hline elevated & 22 & 37.9 & 102 & 30.3 & & 3 & 14.3 & 40 & 31.2 & \\
\hline CA19-9 & & & & & 0.091 & & & & & 0.221 \\
\hline normal & 37 & 63.8 & 254 & 75.4 & & 11 & 52.4 & 88 & 68.8 & \\
\hline elevated & 21 & 36.2 & 83 & 24.6 & & 10 & 47.6 & 40 & 31.2 & \\
\hline
\end{tabular}

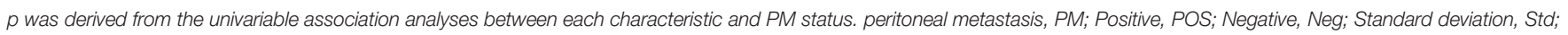
number, N; Upper stomach, U; Middle stomach, M; Lower stomach, L; Duodenum, D; Esophagus, E; carcinoembryonic antigen, CEA; carbohydrate antigen 19-9, CA19-9.

TABLE 2 | Variables and coefficients of the clinical model.

\begin{tabular}{lccc}
\hline Variable & \multicolumn{3}{c}{ Clinic model } \\
\cline { 2 - 4 } & $\boldsymbol{\beta}$ & OR $\mathbf{( 9 5 \%} \mathbf{~ C l )}$ & $\boldsymbol{p}$ \\
\hline Intercept & -2.2351 & - & - \\
L/L+D & -0.7910 & $0.453(0.247-0.832)$ & 0.010 \\
Borrmann type & 1.1324 & $3.103(1.504-6.401)$ & 0.002
\end{tabular}

OR, Odds Ratio; Cl, Confidence interval.

TABLE 3 | Model performance and DeLong's test.

\begin{tabular}{lcc}
\hline Performance & \multicolumn{2}{c}{ Testing cohort } \\
\cline { 2 - 3 } & DCNN model & Clinical model \\
\hline TP & 17 & 18 \\
TN & 112 & 57 \\
FN & 4 & 3 \\
FP & 16 & 71 \\
Sensitivity & 0.810 & 0.857 \\
Specificity & 0.875 & 0.445 \\
AUC & 0.900 & 0.670 \\
& $(0.851-0.953)$ & $(0.615-0.739)$ \\
$p$ & $\backslash$ & $<0.001$ \\
\hline
\end{tabular}

DCNN, deep convolutional neural network; TP, True Positive; TN, True Negative; FN, False Negative; FP, False Positive; AUC, area under curve.

on the testing set subgrouped by sex and age. As shown in Table 4, the DCNN model presented good accuracy in the discrimination of OPM positivity and PM negativity among the different subgroups.

\section{Heatmap Analysis}

Figure 5 shows two representative cases with a cropped GradCAM view superimposed heatmaps on the original CT images. The OPM-positive case 24 was diagnosed by the DCNN model with a prediction score of 0.768 , exhibiting activated status in the highlighting subregions. Furthermore, PM-negative case 88 was diagnosed by the DCNN model with a prediction score of 0.015 , exhibiting inactivated status in all subregions.

\section{Clinical Use}

The decision curve shown in Figure 6 was used to compare the benefit of the DCNN model, all-laparoscopy and no-laparoscopy schemes. We found that if the threshold probability for the clinical decision was less than $80 \%$ (i.e., if the improper surgical procedure for OPM-positive patients was considered more harmful than laparoscopic exploration), the patient would benefit more from the findings of the DCNN model than either the all-laparoscopy or no-laparoscopy schemes.

\section{DISCUSSION}

In this study, we developed a DCNN model to identify OPM in AGC patients prior to surgical treatment. The DCNN model yielded an AUC of 0.900 and sensitivity of $81.0 \%$, which was significantly greater than that of the clinical model (AUC of $0.532, \mathrm{p}<0.001)$. The proposed DCNN model was based on 2D images, focusing on the clinical characteristics of the primary AGC patients identified with OPM. To our knowledge, this is the first deep learning study for OPM detection and diagnosis in AGC patients. 


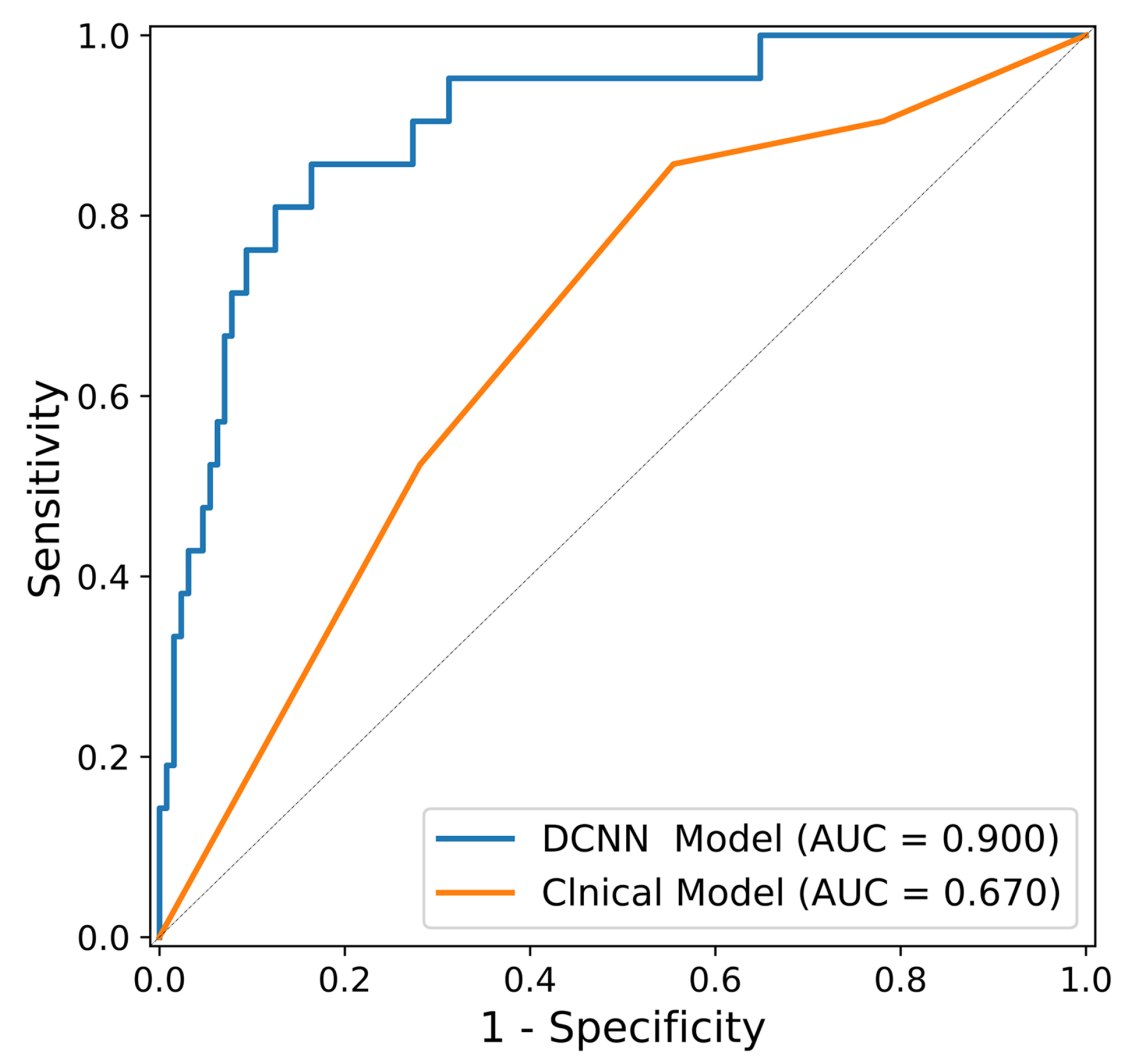

FIGURE 4 | ROC curves of the DCNN model and the clinical model on the testing dataset $(n=149)$.

TABLE 4 | Stratified analysis for DCNN model in the testing cohort.

\begin{tabular}{lccccc}
\hline Performance & \multicolumn{2}{c}{ Age } & \multicolumn{2}{c}{ Gender } & \multicolumn{2}{c}{ Female } \\
\cline { 2 - 4 } & \multicolumn{1}{c}{$\mathbf{6 0}$} & $\mathbf{6 0}$ & 1.000 & 0.889 & 0.833 \\
\hline Sensitivity & 0.800 & 0.718 & 0.902 & 0.862 \\
Specificity & 0.980 & 0.904 & 0.924 & 0.888 \\
AUC & 0.896 & $0.845-0.955$ & $0.851-0.989$ & $0.825-0.956$
\end{tabular}

DCNN, convolutional neural network, AUC, area under curve, Cl, Confidence interval.

Few published papers have focused on the preoperative assessment of PM status in GC patients $(12,14-16,37)$. Previously, CT examination was chosen as the preferable diagnostic imaging modality for PM detection, while MRI and PET/CT were considered secondary choices (38). However, the reported detectability of PM on CT images varied substantially, with an average poor sensitivity of $\sim 50 \%(13,19)$. More recently, Dong et al. reported a radiomics study using CT phenotypes of primary tumors and nearby peritoneum to accurately predict OPM in AGC patients (39). While the concept of "seed and soil", a classic theory of tumor metastasis (40), was applied in Dong's study, the delineated ROI of the peritoneum may not have been representative of the entire "soil" condition. In our study, we focused on the characteristic features of primary tumors and their correlation with a high possibility of OPM to develop a predictive model powered by a DCNN.
Previous studies have shown that clinical factors, including Lauren type, Borrmann type, tumor location and differentiation degree, could be important predictors for PM $(39,41)$. However, preoperative biopsy findings do not typically include the Lauren type and the differentiation degree of GC; these are more often identified in postoperative pathological diagnosis. Therefore, Lauren type and differentiation degree were not included in our study. By incorporating the tumor location and Borrmann type (the independent predictors) in our clinical model, we found that the model had decreased diagnostic accuracy and an AUC of 0.670, suggesting that the involvement of only preoperative clinical features may not be effective for PM prediction in AGC patients. Compared to the clinical model, the proposed DCNN model yielded satisfactory performance and exhibited good generalization ability among patients of different ages and sexes (Table 4). Furthermore, the 


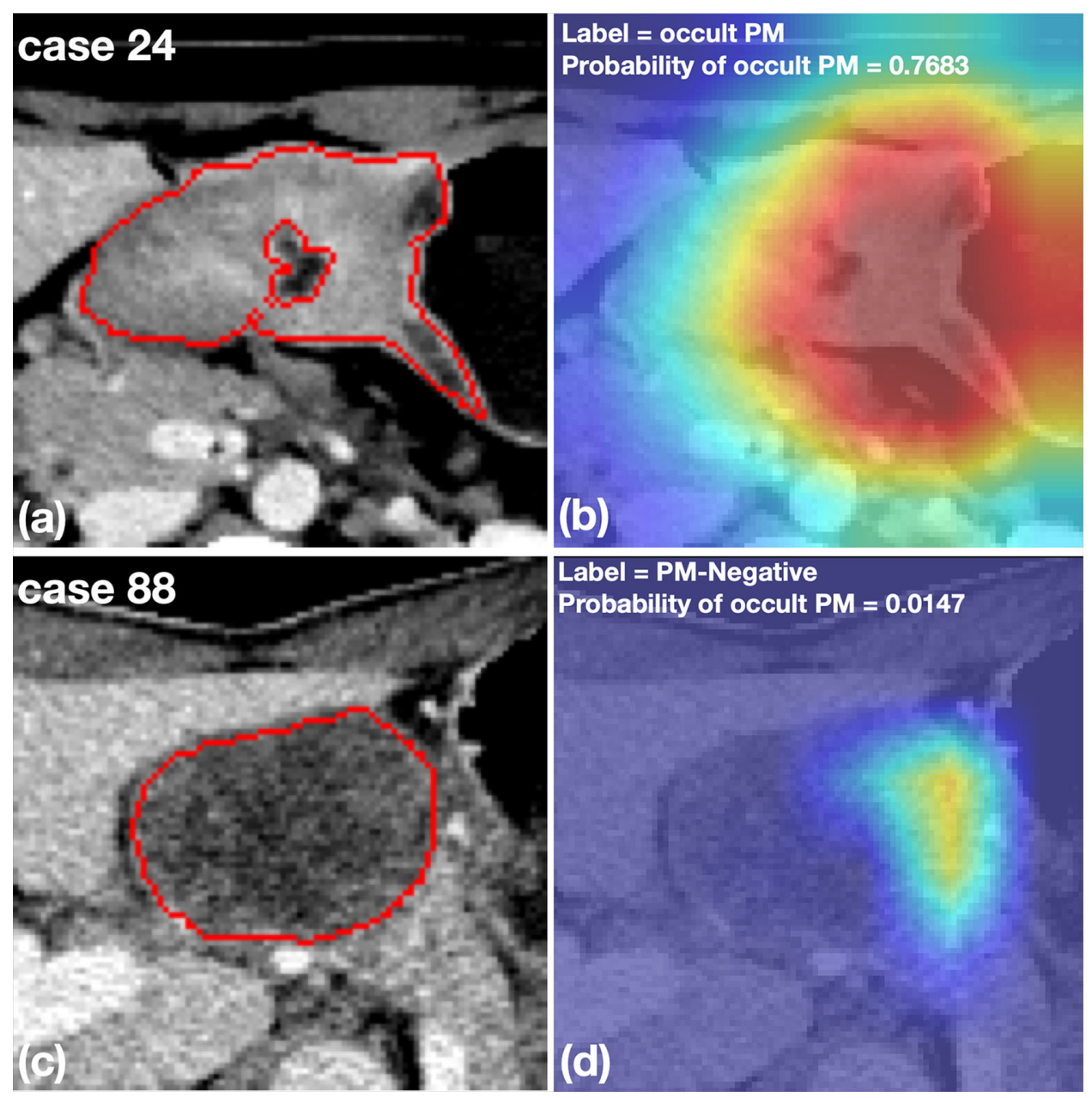

FIGURE 5 | Representative cases with cropped CT images and heatmaps generated by Grad-CAM. (A, B) An OPM-positive patient with pathologically confirmed peritoneal tumor implants during surgery. The DCNN model correctly diagnosed the OPM region with the highest probability of 0.7683 . (C, D) A PM-negative patient who was misclassified by the DCNN model with a probability of OPM of 0.0147 . The subsequent surgery confirmed the patient to have no PM in the peritoneum.

availability of heatmaps (Figure 5) provided a visual display of the PM detection estimated by the DCNN model, which could make it easier for surgeons and oncologists to make clinical assessments accordingly.

The decision of whether to begin surgical treatment in patients with gastric cancer is often a dilemma because of the ambiguity of the PM condition (4). Patients without PM on preoperative CT (but PM positivity at surgery) may undergo an unnecessary surgical procedure due to inadequate preoperative imaging and interpretation. The decision curve analysis (Figure 6) in our study provided an assessment of the value of the DCNN model. For patients with OPM (PMnegative) on conventional CT images, the proposed DCNN model is more suitable than the all-laparoscopy scheme or nolaparoscopy scheme based on the risk of PM. Similarly, if the DCNN model suggested a high possibility of OPM, it would be relatively beneficial to perform diagnostic laparoscopy for confirmation.
Our study indeed has several limitations. First, the delineated ROIs obtained from a single slice (2D) might not be representative of the entire tumor. ROIs extracted from $2 \mathrm{D}$ or 3D images may have an impact on model development and optimization. 3D analysis of the entire tumor is one of our further study interests. Second, we used retrospective datasets to develop the DCNN model and examined a relatively small number of clinical factors. Other factors, such as serological tumor markers, are not initially available on CT scans and may account for any incomplete data. Third, OPM samples were enrolled in the study cohort based on the combined results from initial CT examination (negative) and laparoscopy (positive), which limited the sample size. Finally, external validation is needed to assess the model's diagnostic performance and generalizability across different medical institutions.

In conclusion, compared to a conventional clinical model built using logistic regression, the proposed DCNN model achieved superior diagnostic accuracy for OPM detection and 


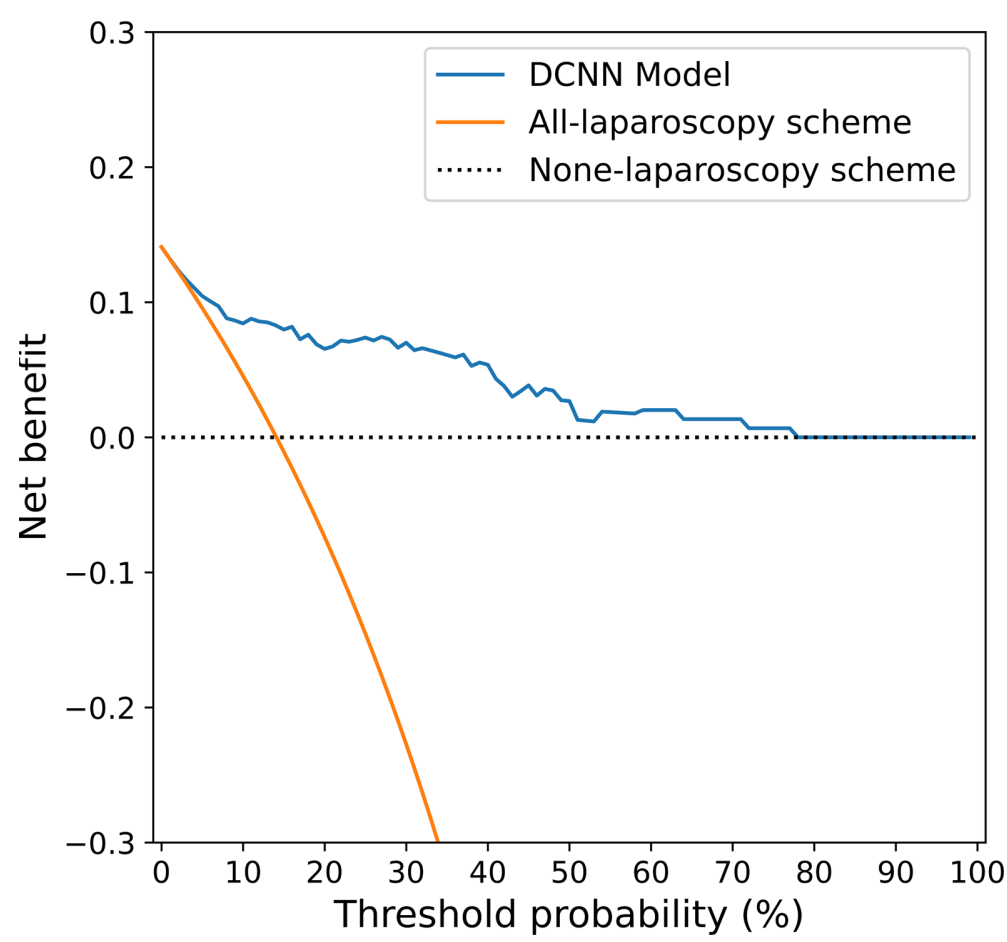

FIGURE 6 | Decision curve analysis for the DCNN model and the all-laparoscopy and no-laparoscopy schemes. Blue line: DCNN prediction model, where the probability of predicting OPM ranges from a minimum of 0 to a maximum of $80 \%$; orange line: all-laparoscopy scheme, assuming all patients should undergo laparoscopy to confirm the presence of OPM; dotted line: no-laparoscopy scheme, assuming no possibility of PM in patients (i.e., the presence of OPM).

diagnoses in AGC patients. The DCNN model may have significant clinical implications for early detection and proper surgical treatment for patients with AGC.

\section{DATA AVAILABILITY STATEMENT}

The original contributions presented in the study are included in the article/Supplementary Material. Further inquiries can be directed to the corresponding authors.

\section{ETHICS STATEMENT}

The studies involving human participants were reviewed and approved by Biomedical Research Ethics Committee of West China Hospital of Sichuan University. Written informed consent for participation was not required for this study in accordance with the national legislation and the institutional requirements.

\section{AUTHOR CONTRIBUTIONS}

$\mathrm{ZH}, \mathrm{JH}$, and BS conceived and designed the study. $\mathrm{ZH}$ drafted the original paper. $\mathrm{DL}, \mathrm{XC}$, and $\mathrm{DH}$ extracted all data and performed the analyses. PY and BL conducted deep learning modeling and statistical analysis based on data. BW, JH, and BS supervised the project and provided direction and guidance throughout the preparation of this manuscript. BS revised the final manuscript. JH provided funding for the study. All authors contributed to the article and approved the submitted version.

\section{FUNDING}

This work was supported by the $1^{\star} 3^{\star} 5$ Project for Disciplines of Excellence, West China Hospital, Sichuan University (no. ZY2017304).

\section{ACKNOWLEDGMENTS}

The authors acknowledge the other members for their contributions to the research, including Jiangfen $\mathrm{Wu}$, Xinyi Fan, Fan Xiang, and Dan Bai.

\section{SUPPLEMENTARY MATERIAL}

The Supplementary Material for this article can be found online at: https://www.frontiersin.org/articles/10.3389/fonc.2020.601869/ full\#supplementary-material 


\section{REFERENCES}

1. Bray F, Ferlay J, Soerjomataram I, Siegel RL, Torre LA, Jemal A. Global cancer statistics 2018: GLOBOCAN estimates of incidence and mortality worldwide for 36 cancers in 185 countries. CA Cancer J Clin (2018) 68:394-424. doi: $10.3322 /$ caac. 21492

2. Coccolini F, Cotte E, Glehen O, Lotti M, Poiasina E, Catena F, et al. Intraperitoneal chemotherapy in advanced gastric cancer. Meta-analysis of randomized trials. Eur J Surg Oncol (2014) 40:12-26. doi: 10.1016/ j.ejso.2013.10.019

3. Thomassen I, van Gestel YR, van Ramshorst B, Luyer MD, Bosscha K, Nienhuijs SW, et al. Peritoneal carcinomatosis of gastric origin: a populationbased study on incidence, survival and risk factors. Int J Cancer (2014) 134:622-8. doi: 10.1002/ijc.28373

4. Kitayama J, Ishigami H, Yamaguchi H, Sakuma Y, Horie H, Hosoya Y, et al. Treatment of patients with peritoneal metastases from gastric cancer. Ann Gastroenterol Surg (2018) 2:116-23. doi: 10.1002/ags3.12060

5. Wang FH, Shen L, Li J, Zhou ZW, Liang H, Zhang XT, et al. The Chinese Society of Clinical Oncology (CSCO): clinical guidelines for the diagnosis and treatment of gastric cancer. Cancer Commun (Lond) (2019) 39:10. doi: 10.1186/s40880-019-0349-9

6. Guner A, Yildirim R. Surgical management of metastatic gastric cancer: moving beyond the guidelines. Transl Gastroenterol Hepatol (2019) 4:58. doi: $10.21037 / \operatorname{tgh} .2019 .08 .03$

7. Coburn N, Cosby R, Klein L, Knight G, Malthaner R, Mamazza J, et al. Staging and surgical approaches in gastric cancer: a clinical practice guideline. Curr Oncol (2017) 24:324-31. doi: 10.3747/co.24.3736

8. Coburn N, Cosby R, Klein L, Knight G, Malthaner R, Mamazza J, et al. Staging and surgical approaches in gastric cancer: A systematic review. Cancer Treat $\operatorname{Rev}(2018)$ 63:104-15. doi: 10.1016/j.ctrv.2017.12.006

9. Muro K, Van Cutsem E, Narita Y, Pentheroudakis G, Baba E, Li J, et al. PanAsian adapted ESMO Clinical Practice Guidelines for the management of patients with metastatic gastric cancer: a JSMO-ESMO initiative endorsed by CSCO, KSMO, MOS, SSO and TOS. Ann Oncol (2019) 30:19-33. doi: 10.1093/ annonc/mdy502

10. Guideline Committee of the Korean Gastric Cancer Association (KGCA). Development Working Group \& Review Panel. Korean Practice Guideline for Gastric Cancer 2018: an Evidence-based, Multi-disciplinary Approach. J Gastric Cancer (2019) 19:1-48. doi: 10.5230/jgc.2019.19.e8

11. Smyth EC, Verheij M, Allum W, Cunningham D, Cervantes A, Arnold D, et al. Gastric cancer: ESMO Clinical Practice Guidelines for diagnosis, treatment and follow-up. Ann Oncol (2016) 27:v38-38v49. doi: 10.1093/ annonc/mdw350

12. Kim SJ, Kim HH, Kim YH, Hwang SH, Lee HS, Park DJ, et al. Peritoneal metastasis: detection with 16- or 64-detector row CT in patients undergoing surgery for gastric cancer. Radiology (2009) 253:407-15. doi: 10.1148/ radiol.2532082272

13. Burbidge S, Mahady K, Naik K. The role of CT and staging laparoscopy in the staging of gastric cancer. Clin Radiol (2013) 68:251-5. doi: 10.1016/ j.crad.2012.07.015

14. Chang DK, Kim JW, Kim BK, Lee KL, Song CS, Han JK, et al. Clinical significance of CT-defined minimal ascites in patients with gastric cancer. World J Gastroenterol (2005) 11:6587-92. doi: 10.3748/wjg.v11. i42.6587

15. Yan TD, Yonemura Y, Morris DL. Surgical strategies for gastric cancer with synchronous peritoneal carcinomatosis. Br J Surg (2007) 94:642; author reply 642-3. doi: 10.1002/bjs.5867. (Br J Surg 2006; 93: 1530-1535).

16. Yajima K, Kanda T, Ohashi M, Wakai T, Nakagawa S, Sasamoto R, et al. Clinical and diagnostic significance of preoperative computed tomography findings of ascites in patients with advanced gastric cancer. Am J Surg (2006) 192:185-90. doi: 10.1016/j.amjsurg.2006.05.007

17. Marcus C, Subramaniam RM. PET/Computed Tomography and Precision Medicine: Gastric Cancer. PET Clin (2017) 12:437-47. doi: 10.1016/ j.cpet.2017.05.004

18. Lim JS, Yun MJ, Kim MJ, Hyung WJ, Park MS, Choi JY, et al. CT and PET in stomach cancer: preoperative staging and monitoring of response to therapy. Radiographics (2006) 26:143-56. doi: 10.1148/rg.261055078
19. Wang Z, Chen JQ. Imaging in assessing hepatic and peritoneal metastases of gastric cancer: a systematic review. BMC Gastroenterol (2011) 11:19. doi: 10.1186/1471-230X-11-19

20. Ramos RF, Scalon FM, Scalon MM, Dias DI. Staging laparoscopy in gastric cancer to detect peritoneal metastases: A systematic review and meta-analysis. Eur J Surg Oncol (2016) 42:1315-21. doi: 10.1016/j.ejso.2016.06.401

21. Sugarbaker PH. Preoperative Assessment of Cancer Patients with Peritoneal Metastases for Complete Cytoreduction. Indian J Surg Oncol (2016) 7:295302. doi: 10.1007/s13193-016-0518-0

22. Hamet P, Tremblay J. Artificial intelligence in medicine. Metabolism (2017) 69S:S36-36S40. doi: 10.1016/j.metabol.2017.01.011

23. Huang S, Yang J, Fong S, Zhao Q. Artificial intelligence in cancer diagnosis and prognosis: Opportunities and challenges. Cancer Lett (2020) 471:61-71. doi: 10.1016/j.canlet.2019.12.007

24. Cuocolo R, Caruso M, Perillo T, Ugga L, Petretta M. Machine Learning in oncology: A clinical appraisal. Cancer Lett (2020) 481:55-62. doi: 10.1016/ j.canlet.2020.03.032

25. Hosny A, Parmar C, Quackenbush J, Schwartz LH, Aerts H. Artificial intelligence in radiology. Nat Rev Cancer (2018) 18:500-10. doi: 10.1038/s41568-018-0016-5

26. Mayo RC, Leung J. Artificial intelligence and deep learning - Radiology's next frontier. Clin Imaging (2018) 49:87-8. doi: 10.1016/j.clinimag.2017.11.007

27. Deig CR, Kanwar A, Thompson RF. Artificial Intelligence in Radiation Oncology. Hematol Oncol Clin North Am (2019) 33:1095-104. doi: 10.1016/ j.hoc.2019.08.003

28. Wang H, Zhou Z, Li Y, Chen Z, Lu P, Wang W, et al. Comparison of machine learning methods for classifying mediastinal lymph node metastasis of nonsmall cell lung cancer from 18F-FDG PET/CT images. EJNMMI Res (2017) 7:11. doi: 10.1186/s13550-017-0260-9

29. Ehteshami Bejnordi B, Veta M, Johannes van Diest P, van Ginneken B, Karssemeijer N, Litjens G, et al. Diagnostic Assessment of Deep Learning Algorithms for Detection of Lymph Node Metastases in Women With Breast Cancer. JAMA (2017) 318:2199-210. doi: 10.1001/jama.2017.14585

30. Liu K, Chen XZ, Zhang WH, Zhang DY, Luo Y, Yu Y, et al. "Four-Step Procedure" of laparoscopic exploration for gastric cancer in West China Hospital: a retrospective observational analysis from a high-volume institution in China. Surg Endosc (2019) 33:1674-82. doi: 10.1007/s00464-018-6605-2

31. Wong SC, Gatt A, Stamatescu V, McDonnell MD. Understanding data augmentation for classification: when to warp. In: 2016 International Conference on Digital Image Computing: Techniques and Applications. Institute of Electrical and Electronics Engineers (2016). p. 59-64.

32. Chollet F. Xception: Deep Learning with Depthwise Separable Convolutions [arXiv]. arXiv (2016) 14:pp-14. doi: 10.1109/CVPR.2017.195

33. Russakovsky O, Deng J, Su H, Krause J, Satheesh S, Ma S, et al. ImageNet Large Scale Visual Recognition Challenge. Int J Comput Vision (2015) 115:211-52. doi: 10.1007/s11263-015-0816-y

34. Kim DH, MacKinnon T. Artificial intelligence in fracture detection: transfer learning from deep convolutional neural networks. Clin Radiol (2018) 73:43945. doi: 10.1016/j.crad.2017.11.015

35. Wolpert DH. STACKED GENERALIZATION. Neural Netw (1992) 5:241-59. doi: 10.1016/S0893-6080(05)80023-1

36. Sakamoto Y, Kitagawa G. Akaike information criterion statistics. Dordrecht, The Netherlands: Kluwer Academic Publishers (1987).

37. Kim SH, Choi YH, Kim JW, Oh S, Lee S, Kim BG, et al. Clinical significance of computed tomography-detected ascites in gastric cancer patients with peritoneal metastases. Med (Baltimore) (2018) 97:e9343. doi: 10.1097/ MD.0000000000009343

38. Laghi A, Bellini D, Rengo M, Accarpio F, Caruso D, Biacchi D, et al. Diagnostic performance of computed tomography and magnetic resonance imaging for detecting peritoneal metastases: systematic review and metaanalysis. Radiol Med (2017) 122:1-15. doi: 10.1007/s11547-016-0682-x

39. Dong D, Tang L, Li ZY, Fang MJ, Gao JB, Shan XH, et al. Development and validation of an individualized nomogram to identify occult peritoneal metastasis in patients with advanced gastric cancer. Ann Oncol (2019) 30:431-8. doi: 10.1093/annonc/mdz001

40. Akhtar M, Haider A, Rashid S, Al-Nabet A. Paget's "Seed and Soil” Theory of Cancer Metastasis: An Idea Whose Time has Come. Adv Anat Pathol (2019) 26:69-74. doi: 10.1097/PAP.0000000000000219 
41. Kim M, Jeong WK, Lim S, Sohn TS, Bae JM, Sohn IS. Gastric cancer: development and validation of a CT-based model to predict peritoneal metastasis. Acta Radiol (2020) 61(6):732-42. doi: 10.1177/0284185119882662. 284185119882662

Conflict of Interest: The authors declare that the research was conducted in the absence of any commercial or financial relationships that could be construed as a potential conflict of interest.
Copyright (C) 2020 Huang, Liu, Chen, He, Yu, Liu, Wu, Hu and Song. This is an openaccess article distributed under the terms of the Creative Commons Attribution License (CC BY). The use, distribution or reproduction in other forums is permitted, provided the original author(s) and the copyright owner(s) are credited and that the original publication in this journal is cited, in accordance with accepted academic practice. No use, distribution or reproduction is permitted which does not comply with these terms. 